\title{
Study on malachite green residue detection system
}

\author{
Tong Shen, Tong Zhou*, Ying Wan, and Yan Su \\ School of Mechanical Engineering, Nanjing University of Science and Technology, Nanjing, China
}

\begin{abstract}
In this work, a malachite green residue detection system for aquaculture monitoring was established. The system includes a new method based on DNA adapter body of malachite green sensor, a handheld electrochemical detector, and the combination of the two, which constitute a set of high performance, convenient and portable malachite green residue detection system. According to the experimental results, the detection of this system is range from $1.0 \times 10^{-3} \mu \mathrm{g} \cdot \mathrm{mL}^{-1}$ to $10 \mu \mathrm{g} \cdot \mathrm{mL} \mathrm{L}^{-1}$. Hence, the detection system proposed in this paper can meet the accuracy, convenience and quick detection of malachite green residue in aquatic products water.
\end{abstract}

\section{Introduction}

Malachite green (MG), a bactericidal dye, has been widely used in commercial aquaculture and aquatic transportation because of its special effects on the water mildew of the fish and fish eggs [1]. However, MG has the effect of carcinogenic and teratogenicity [2], and it can cause harm to the human body through the food chain. Therefore, the European Union has set a detection limit [3] for MG at a level of $2 \mathrm{mg} \mathrm{kg}^{-1}$. The department of agriculture has also banned such antibiotics.

Nowadays, electrochemical detection method [4] has been widely used in the detection of antibiotics because of its high detection accuracy, strong analytical capability and high detection speed. Aptamer [5,6], with the advantages of high sensitivity and good selectivity, is widely used in the establishment of electrochemical sensors. Using the aptamer sensor can be more efficiently to identify testing material, Therefore, in this paper, a method of electrochemical detection using aptamer is established.

However, due to the electrochemical apparatus is large in volume and high in cost, and using electrochemical apparatus for testing is not convenient [7], it's of vital significance to design a kind of portable handheld electrochemical.

Herein, this paper has established a hand-held electrochemical detector, which can detect MG rapidly. It includes biosensors based on DNA adaptors and hand-held electrochemical apparatus, and users can control the detection by the electrochemical apparatus and make the test results displayed in the mobile APP. This detection strategy has the advantages of low cost, sensitive detection, high detection speed, simple operation and easy to carry.

\footnotetext{
* Corresponding author: zhoutong@njust.edu.cn
} 


\section{Design of malachite green sensor}

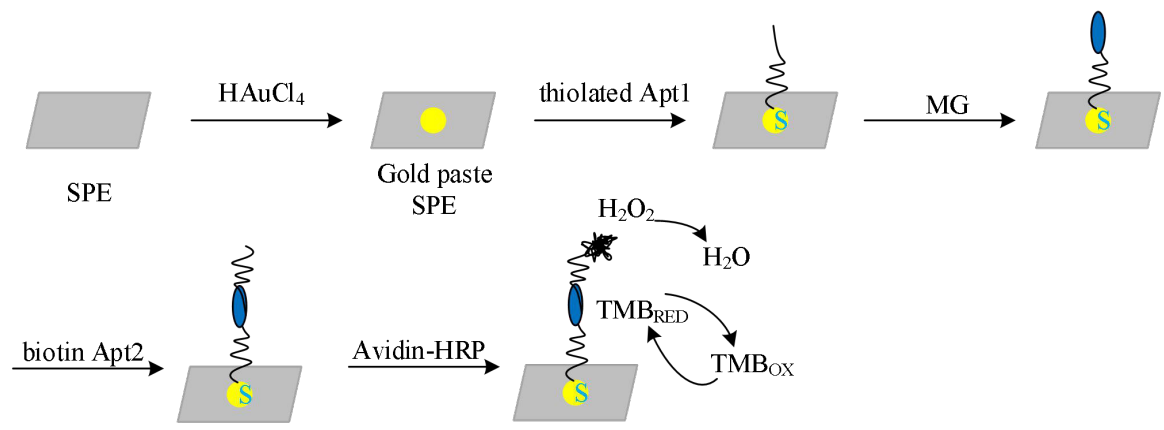

Fig. 1. Schematic diagram of the construction of electrochemical sensor based on DNA adaptor.

The construction process of electrochemical sensor based on DNA adaptor is shown in figure 1. First, the screen printing electrode (SPE) was cleaned with ultra-pure water for 1 min. The nanoparticles were then modified to the surface of the carbon electrode (the working electrode), and the apt 1 with malachite green will be more stable on the electrode surface. The specific steps are as follows: An electrode was scanned in a $0.5 \mathrm{M}$ dilute $\mathrm{H}_{2} \mathrm{~S}_{4}$ by a $\mathrm{CV}$ scan. The initial potential is $-0.2 \mathrm{~V}$, the highest potential is $1.5 \mathrm{~V}$, and the termination potential is $-0.2 \mathrm{~V}$. The scan is performed for one cycle and the $\mathrm{CV}$ scan curve is obtained. Then, the electrodes were washed with PBS buffer for $1 \mathrm{~min}$ and then dried. Finally, the time current method was employed to electrodes in the $1 \mathrm{mg} / \mathrm{mL} \mathrm{HAuCl}_{4}$ solution, the scanning voltage was $-0.2 \mathrm{~V}$, the scanning time was $100 \mathrm{~s}$. and the electrodes were washed three times with PBS buffer after the scanning.

Apt1 of MG at appropriate concentrations of $3 \mu \mathrm{L}$ were then added to each electrode for overnight at $4{ }^{\circ} \mathrm{C}$, waiting for the Aptl fixed on the electrode. After fixation, rinsed with PBS buffer for 1min, removing the specific adsorption.

After finishing the modification of the adaptor1, different concentrations of MG were added to the working electrode. and then $3 \mu \mathrm{L}$ bio-Apt 2 at $1 \mu \mathrm{M}$ was added to each electrode. The Apt1, MG and Apt2 formed a "sandwich" structure. After this, rinsed with PBS buffer for $1 \mathrm{~min}$, removing the specific adsorption.

After being washed, electrodes were treated with Av-HPR. After 20-min incubation at room temperature, electrodes were washed with PBS buffer $(\mathrm{pH} 7.4)$ and subjected to electrochemical measurements. Av-HRPs attached to the biotin labels via the biotin-avidin bridge, which could be transuded to the catalytic amperometric readout.

Finally, cyclic voltammetry (CV) [8] and amperometry [9] were employed on the printing electrodes in the TMB solution with $\mathrm{H}_{2} \mathrm{O}_{2}$ to find response current. The scanning current was calculated in the concentration-current relation curve.

\section{Hardware design of malachite green detector}

\subsection{The overall hardware structure of the detector}

The hardware design of malachite green detector includes scanning voltage generation circuit, constant potential circuit, weak current detection circuit, data acquisition circuit, MCU main control circuit and power module. At the last, the Bluetooth module sends the data to the phone. And this Malachite green detector chose the STM32F103RBT6 of ST company as the main control chip. See figure 2. 


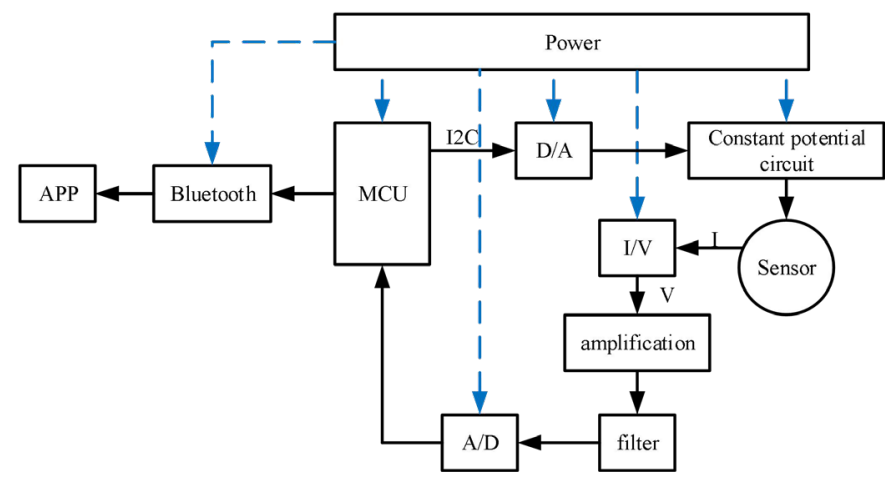

Fig. 2. The overall structure of the detector hardware.

\subsection{Constant potential circuit}

In the three-electrode system [10], as the chemical reaction progresses, the concentration of the reactants on the surface of the electrode decreases, and the concentration of the products increases continuously, the electric potential between the working electrode and the reference electrode also deviates from the initial set voltage.

According to the input and output characteristics of the reverse proportional amplifier, the voltage of the reference electrode can be obtained equation (1):

$$
V_{\mathrm{RE}}-2.5=-\frac{\mathrm{R}_{2}}{\mathrm{R}_{3}}\left(V_{\mathrm{DAC}}-2.5\right)=-\left(V_{\mathrm{DAC}}-2.5\right)
$$

Therefore, the potential between the working voltage and the reference voltage is equation (2):

$$
V_{\mathrm{WE}}-V_{\mathrm{RE}}=2.5-V_{\mathrm{RE}}=V_{\mathrm{DAC}}-2.5
$$

Therefore, the voltage constant between the working electrode and the reference electrode is controlled by controlling the output voltage of the DAC through the signal generation circuit.

\subsection{Weak current detection circuit}

The malachite green sensor designed in this paper is a kind of current type sensor, and because the measurement concentration requirement is accurate, the generated current value and current change value are also relatively weak. Therefore, the detection of weak current is very important. This paper adopts the principle of reverse current amplification circuit to design its weak current detection circuit, and uses LMP7721 as the operation and release of Microsoft current amplifying circuit. According to the principle of reverse current amplification, the amplification voltage can be calculated as equation (3):

$$
V_{\mathrm{OUT} 1}=I_{\mathrm{WE}} \cdot \mathrm{R}+2.5
$$

\subsection{Waveform scanning circuit}

In this paper, DAC selects 16 bits of Analog Device: DAC8571. The single-chip microcomputer control DAC8571 output voltage waveform curve through $\mathrm{I}^{2} \mathrm{C}$ communication interface.

The $A_{0}$ is selected for the device address, in this paper, $A_{0}$ is connected to the ground. In addition, the relationship between output voltage value and digital quantity of DAC8571 is equation (4): 


$$
D A T A=\frac{\mathrm{V}_{\mathrm{DAC}} \cdot 2^{16}}{5}
$$

The accuracy of the reference voltage affects the accuracy of the scanning voltage directly, so a stable voltage chip should be selected to provide a stable reference voltage for DAC. Because the ADR445 has the characteristics of low noise and long-term stability, this paper selects the ADR445 of Analog Device company to provide the accurate $5 \mathrm{~V}$ reference voltage for DAC8571.

\subsection{Data acquisition circuit}

In this paper, the requirements of the data acquisition system of the input voltage range from $0-2.5 \mathrm{~V}$, the sensor output current of the minimum current variation in the level of $10 \mathrm{pA}$, the voltage change obtained by the current amplification module is about $0.05 \mathrm{mV}$, and the known reference voltage value, $\mathrm{V}_{\mathrm{REF}+}$ is $2.5 \mathrm{~V}$, so the precision of the corresponding 16-bit $\mathrm{ADC}$ is equation (5):

$$
\mathrm{s}=\frac{2.5}{2^{16}-1} \approx 3.81 \times 10^{-2} \mathrm{mV} / \mathrm{bit}
$$

Because $3.8 \times 10^{-2} \mathrm{mV}$ is larger than $0.05 \mathrm{mV}, 16$ bits of ADC are selected for the data collection of the system; Finally chooses the Analog Devices Inc., a chip of a 64 pin high precision, and successive approximation (SAR) of group type 16 bits D/A conversion chip:AD7656. (6):

The sample data of AD7656 and the corresponding voltage value relation is equation

$$
V_{\text {in }}=\frac{D A T A}{0 \mathrm{xFFF}} \cdot 2 \cdot \mathrm{V}_{\mathrm{REF}+}
$$

In this formula, $V_{\text {in }}$ represents the analog input voltage, DATA represents the sampled data, and $\mathrm{V}_{\mathrm{REF}+}$ represents the input reference voltage.

\section{The software design of the detector}

This article detector software design is the MCU program design, mainly includes the D/A output waveform procedures, data collection procedures, serial communication program, etc., At the last phone receiving data and display the preservation.

\subsection{Overall design of program}

The overall flow of the program is shown in figure 3. After the system is powered on, the mobile phone send "start" command to MCU, then MCU sends scanning waveform of cyclic voltammetry and time scanning, and continuous real-time acquisition of the sensor generated by the current value. After the scanning, the concentration of the detector was determined by the CV relation., the final data will be calculated to be displayed and saved by mobile phone via Bluetooth.

\subsection{Program design of waveform scanning}

In this paper, the $\mathrm{I}^{2} \mathrm{C}$ interface of STM32F103 is selected as the $\mathrm{I}^{2} \mathrm{C}$ communication mode to communicate with DAC8571, and the program flow chart of cyclic voltammetry scan is shown in figure 4 . The timer of STM32F103 is used to set the timer to be interrupted once $100 \mathrm{~ms}$, changing the voltage value of $\mathrm{D} / \mathrm{A}$ output, and collecting the changed current of the 
sensor current. The scanning time of this triangle wave of the experiment was $14 \mathrm{~s}$, and the output period was $14 \mathrm{~s}$ and the amplitude was $0.7 \mathrm{~V}$.

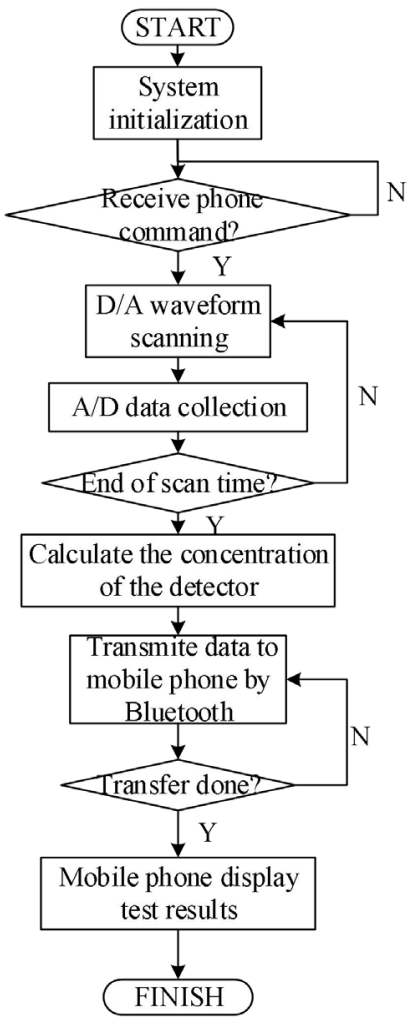

Fig. 3. Overall flow chart of the program.

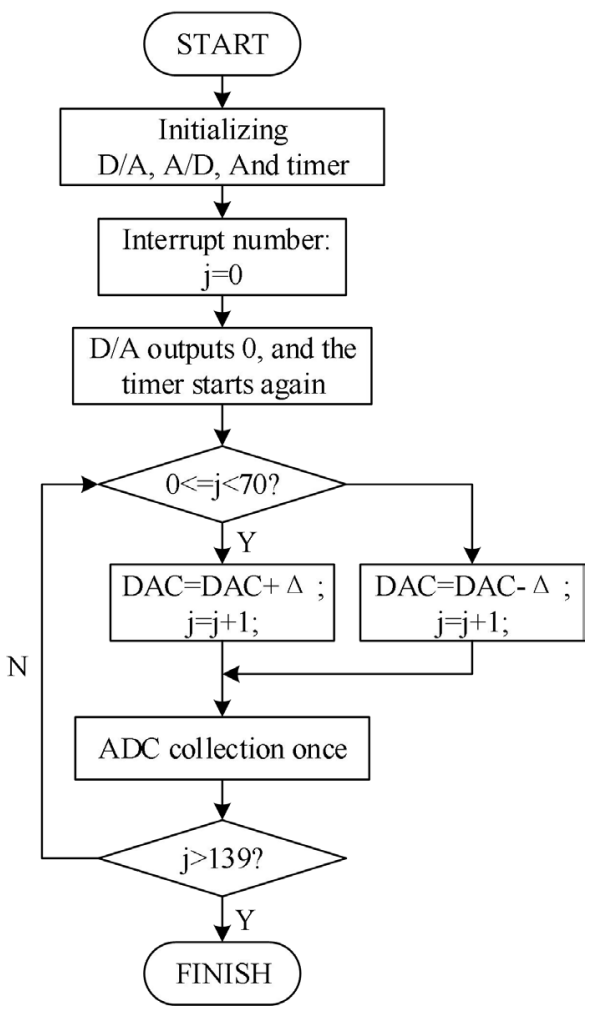

Fig. 4. Flow chart of cyclic voltammetry.

Before starting the conversion, giving the device a RESET pulse for 100ns, then gives the CONVST a rising edge to start the synchronous conversion. When the BUSY signal is detected from high level to low power, the conversion is finished. Therefore, it is possible to judge whether the data transformation is over by judging the drop edge of the BUSY signal. So that the data can be read out immediately at the end of each transition.

\section{Experimental results and discussion}

Using the detection system in this paper to detect malachite green content in water. Adding the standard concentration of malachite green to the water sample, using this test system to measure malachite green content in water, the comparison results are shown in table 1. It can be seen from the table that the measurement method and relative error of the measurement system are controlled within $10 \%$, which can be used for the measurement of malachite green residue concentration in more complex water quality.

Table 1. Analysis of MG test results in experimental samples.

\begin{tabular}{|c|c|}
\hline Standard value $\left(\mu \mathrm{g} \cdot \mathrm{mL}^{-1}\right)$ & The determination of value $\left(\mu \mathrm{g} \cdot \mathrm{mL}^{-1}\right)$ \\
\hline $1.000 \times 10^{-3}$ & $(0.946 \pm 0.045) \times 10^{-3}$ \\
\hline $1.000 \times 10^{-2}$ & $(1.053 \pm 0.049) \times 10^{-2}$ \\
\hline $1.000 \times 10^{-1}$ & $(1.028 \pm 0.052) \times 10^{-1}$ \\
\hline 1.000 & $0.985 \pm 0.050$ \\
\hline
\end{tabular}




\section{Conclusion}

To sum up, this paper constructs an electrochemical biosensor based on DNA adaptor, and a set of hand-held electrochemical detector. Among them, the nanoparticles were modified on the surface of carbon electrode by electrochemical deposition. The results show that the constructed detection system has a wide linear range of $1.0 \times 10^{-3} \mu \mathrm{g} \cdot \mathrm{mL}^{-1}$ to $10 \mu \mathrm{g} \cdot \mathrm{mL}^{-1}$. The minimum detection limit of $1.0 \times 10^{-3} \mu \mathrm{g} \cdot \mathrm{mL}^{-1}$. The system can measure the malachite green content in fishing water efficiently and accurately, easy to carry and easy to operate. Therefore, this detection system provides a new method for detection of malachite green concentration for accurate and rapid detection.

\section{References}

1. Feng X, Ning G, Zhang H, et al. A novel "dual-potential" electrochemiluminescence aptasensor array using CdS quantum dots and luminol-gold nanoparticles as labels for simultaneous detection of malachite green and chloramphenicol[J]. Biosensors \& Bioelectronics, 74:587-593 (2015)

2. Wang H, Wang Y, Liu S, et al. An RNA aptamer-based electrochemical biosensor for sensitive detection of malachite green[J]. Rsc Advances, 4(105):60987-60994 (2014)

3. Zhu D, Li Q, Pang X, et al. A sensitive electrochemical impedance immunosensor for determination of malachite green and leucomalachite green in the aqueous environment[J]. Analytical \& Bioanalytical Chemistry, 408(20):5593-5600 (2016)

4. Zhu L, Santiagoschübel B, Xiao H, et al. Electrochemical oxidation of fluoroquinolone antibiotics: Mechanism, residual antibacterial activity and toxicity change.[J]. Water Research, 102:52-62 (2016)

5. Wan Y, Wang P, Su Y, et al. Ultrasensitive electrochemical DNA sensor based on the target induced structural switching and surface-initiated enzymatic polymerization.[J]. Biosensors \& Bioelectronics, 55(9):231-236 (2014)

6. Rowe A A, Miller E A, Plaxco K W. Reagentless Measurement of Aminoglycoside Antibiotics in Blood Serum via an Electrochemical, Ribonucleic Acid Aptamer-Based Biosensor[J]. Analytical Chemistry, 82(17):7090-5 (2010)

7. Pinacho D G, Sánchez-Baeza F, Pividori M I, et al. Electrochemical Detection of Fluoroquinolone Antibiotics in Milk Using a Magneto Immunosensor[J]. Sensors, 14(9):15965-15980 (2014)

8. Nicholson R S. Theory and Application of Cyclic Voltammetry for Measurement of Electrode Reaction Kinetics.[J]. Analytical Chemistry, 37(11):1351-1355 (1965)

9. Huang L, Shen H, Atkinson M A, et al. Detection of exocytosis at individual pancreatic beta cells by amperometry at a chemically modified microelectrode[J]. Proceedings of the National Academy of Sciences of the United States of America, 92(21):9608 (1995)

10. Suzuki H, Hirakawa T, Sasaki S, et al. An integrated three-electrode system with a micromachined liquid-junction $\mathrm{Ag} / \mathrm{AgCl}$ reference electrode[J]. Analytica Chimica Acta, 387(1):103-112 (1999) 